\title{
AUTO SINTONIA UTILIZANDO MODELO ARMAX
}

\author{
A. U. A. JÚNIOR ${ }^{1}$, M. M. SILVA ${ }^{1}$, A. C. NASCIMENTO ${ }^{1}$, H. BISPO ${ }^{1}$ e J. N. SILVA ${ }^{1}$ \\ ${ }^{1}$ Universidade Federal de Campina Grande, Unidade Acadêmica de Engenharia Química \\ E-mail para contato: nilton@ deq.ufcg.edu.br
}

\begin{abstract}
RESUMO - Este trabalho apresenta um método de auto-sintonia de controladores PID (Proporcional, Integral e Derivativo), utilizando o modelo ARMAX (AutoRegressive Moving Average with eXogeneus variable) junto ao método relé para a estimação dos parâmetros de sintonia. Tal procedimento foi implementado em plataforma VBA (Visual Basic for Application), para controle de uma coluna de destilação numa unidade de tratamento de nafta modelada em Aspen Dynamics ${ }^{\circledR}$, a mesma previamente validada com dados reais de planta. Para estimação on-line dos parâmetros do modelo ARMAX foi utilizado o método dos mínimos quadrados estendido com predição de erros. Aplicando um estímulo no modelo ARMAX a partir do relé, os parâmetros de ganho final e período final puderam ser calculados, o que possibilitou a obtenção dos parâmetros de sintonia em malha fechada de acordo com as equações clássicas de sintonia de ZieglerNichols. A estratégia utilizada para correção on-line dos parâmetros dos controladores apresentou desempenho satisfatório na malha de pressão quanto à redução do efeito de distúrbio na planta e modificação do ponto de operação.
\end{abstract}

\section{INTRODUÇÃO}

Controlar um processo significa atuar no mesmo com o intuito de obter resultados desejados dentro de limites de tolerância. Sempre que houver um distúrbio ou variação de carga no processo, a variável controlada deve retornar ao ponto de operação estabelecido, dentro do tempo prescrito e com um erro limitado. O controlador é o elemento que executa o algoritmo de controle e é capaz de detectar os erros infinitesimais entre o valor da variável de processo e o ponto de ajuste e, responder, instantaneamente, de acordo com os modos de controle e seus ajustes. O uso de controladores é necessário para manter o processo sob controle. Neste campo, controladores PID possuem expressiva aplicação devido à sua metodologia simples e bastante difundida no setor industrial.

Para manter o bom funcionamento do controle de um processo, no entanto, é necessária a sintonia correta dos controladores a fim de que as especificações do set-point sejam alcançadas para as variáveis controladas. Desde as técnicas pioneiras propostas por ZieglerNichols em 1942, uma vasta quantidade de pesquisas foi desenvolvida neste setor. Com base nas técnicas de sintonia de Ziegler-Nichols, estudos introduzidos por Åström e Hagglünd (1984) propuseram a metodologia de sintonia automática de controladores.

A auto-sintonia de controladores é capaz de reajustar automaticamente os parâmetros de sintonia de controladores do processo. É reconhecido que na prática muitos controladores industriais são insatisfatoriamente sintonizados e, por esta razão, as técnicas de sintonia automática têm recebido cada vez mais atenção dos pesquisadores e engenheiros de controle. 
Neste sentido, a auto-sintonia é uma área bastante promissora e que tem uma grande oportunidade de aplicação em diversos processos químicos com o intuito de melhorar a controlabilidade destes.

Este trabalho tem como objetivo implementar um sistema de auto-sintonia de controladores do tipo PID, utilizando o modelo auto-regressivo ARMAX como modelo do processo. Para testar o sistema de auto-sintonia proposto, uma unidade de tratamento de nafta foi utilizada, sendo a mesma modelada na plataforma Aspen Dynamics ${ }^{\circledR}$, previamente validada com dados reais de planta.

\section{REVISÃO BIBLIOGRÁFICA}

\subsection{Auto-sintonia}

Em 1942, Ziegler e Nichols sugeriram duas abordagens para ajustar os parâmetros de um controlador PID. O primeiro método, o método da malha fechada, baseia-se em testar a planta com o controlador PID, por tentativa e erro até serem determinados os parâmetros de ganho final e período final para posterior cálculo dos parâmetros de sintonia. O segundo é em malha aberta e análise realizada a partir da curva de reação do processo assumido como sendo de primeira ordem (SILVA, 2013).

Åström e Hägglund (1988) identificaram as limitações da metodologia pioneira de sintonia proposta por Ziegler-Nichols e a aperfeiçoaram propondo metodologia de autosintonia através de um ensaio de identificação do ponto crítico utilizando um relé realimentado. Dentre as principais vantagens da metodologia proposta destacam-se: a simplicidade de realização do ensaio, a rapidez na sintonia reduzindo custos de projeto e principalmente o fato do método operar em malha fechada não interferindo de forma muito significativa na rotina do processo que se deseja controlar. Diante de tais vantagens e percebendo a potencialidade da auto-sintonia, diversos pesquisadores têm desenvolvido pesquisas buscando melhorar o método proposto.

A estratégia clássica de auto-sintonia proposta em estudos desenvolvidos por Åström (1996) é realizada com o relé ligado ao processo conforme demonstrado na Figura 1, onde o mesmo substitui um controlador PID, o qual estimula o processo. A grande desvantagem dessa estratégia é que o processo não permanece controlado durante o tempo de ajuste. Assim, pode acarretar prejuízos ao processo pelo fato do sistema poder operar fora dos limites de segurança das condições operacionais de equipamentos, assim também como a qualidade do produto final pode apresentar características insatisfatórias para as especificações do processo, e, assim, gerar prejuízo financeiro.

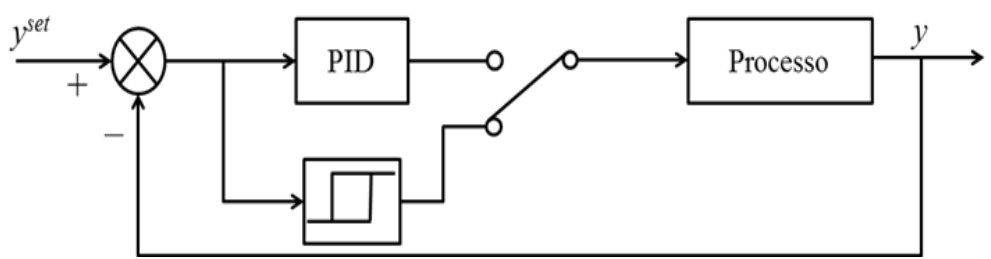

Figura 1 - Estratégia clássica de auto-sintonia. 
Para superar esta dificuldade uma pequena modificação foi considerada por Silva (2013), em que ao invés de substituir o controlador, o relé estimula o modelo identificado trabalhando em paralelo ao processo. Essa metodologia modificada faz com que o relé esteja simultaneamente com a ação do controlador PID. Verifica-se na Figura 2 a estrutura detalhada adotada por Silva (2013) para a geração automática dos parâmetros de sintonia para o controlador PID em paralelo com a execução do processo. O diferencial das modificações é a etapa de identificação do processo por método recursivo e estimação pelo modelo ARMAX, com isso permitindo o ajuste dos parâmetros de sintonia do controlador, com este em operação durante todo o procedimento.

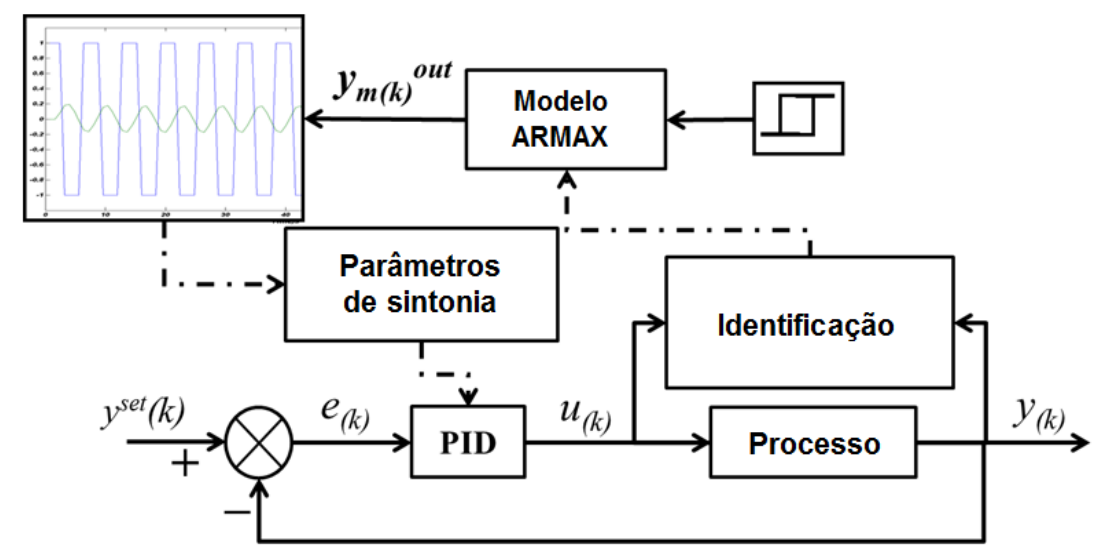

Figura 2 - Estrutura de auto-sintonia modificada.

\subsection{Modelagem do processo}

A modelagem matemática de sistemas é uma metodologia muito comum em ciência e na engenharia devido a sua importância para extrair conhecimento sobre sistema por meio de simulações. No presente trabalho aplicou-se o modelo ARMAX (Modelo Auto-regressivo com média móvel e entrada exógena), conforme apresentado na Equação 1.

$A(q) y(t)=B(q) u(t)+C(q) e(t)$

O modelo geral utilizado para representar a relação entre a entrada e saída do sistema com aplicação da média móvel à perturbação é dado por:

$$
\begin{aligned}
y_{(t)}+a_{1} y_{(t-1)}+a_{2} y_{(t-2)} & +\ldots+a_{n_{a}} y_{\left(t-n_{a}\right)}=b_{1} u_{(t-1)}+b_{2} u_{(t-2)}+\ldots \\
& +b_{n_{b}} u_{\left(t-n_{b}\right)}+c_{1} e_{(t-1)}+c_{2} e_{(t-2)}+\ldots+c_{n c} e_{\left(t-n_{c}\right)}+e(t)
\end{aligned}
$$

Para a determinação dos parâmetros do modelo ARMAX foi considerado um vetor parâmetros $(\theta)$, conforme apresentado na Equação 3 .

$\theta=\left[a_{1}, a_{2}, \ldots, a_{n_{a}} ; b_{1}, b_{2}, \ldots, b_{n_{b}} ; c_{1}, c_{2}, \ldots, c_{n_{c}}\right]^{T}$ 
Além disso, foi inserido um vetor regressão para armazenar a variável de saída, variável de entrada e desvio do processo. Este vetor segue expresso na Equação 4.

$$
\varphi(t, \theta)=\left[-y_{t-1},-y_{t-2}, \ldots,-y_{t-n_{a}} ; u_{t-1}, u_{t-2}, \ldots, u_{t-n_{b}} ; e_{(t-1, \theta)}, e_{(t-2, \theta)}, \ldots, e_{\left(t-n_{c}, \theta\right)}\right]^{T}
$$

Assim, pode ser calculado o vetor $\hat{y}(t / \theta)$, que representa o valor preditivo da variável de saída $(y)$ no instante $t$ utilizando o vetor parâmetros $\theta$. A correlação para a determinação desse vetor segue apresentada na Equação 5.

$\hat{y}(t / \theta)=\varphi^{T}{ }_{(t, \theta)} \theta$

Para a determinação do vetor $\theta$ aplicou-se o algoritmo dos mínimos quadrados recursivo conforme proposto por Ljung e Söderström (1983) e Holst (1977). O método dos mínimos quadrados é comumente usado no sistema de identificação. Seu principio é que o parâmetro desconhecido de um modelo matemático deve ser escolhido por minimização da soma dos quadrados da diferença entre os valores observados e os valores de saída. Assim, os parâmetros estimados com uso do algoritmo mínimos quadrados recursivo $\left(\theta^{*}\right)$ pode ser expresso, conforme apresentado na Equação 6, por:

$\theta^{*}=\left[\varphi_{(t)}^{T} \varphi_{(t)}\right]^{-1} \varphi_{(t)}^{T} y_{(t)}$

A ideia fundamental da estimação é a comparação da resposta observada do sistema $(y(t))$ com a saída do modelo parametrizado $(\hat{y}(t / \theta))$ cuja estrutura é a mesma que a do modelo da planta. Em seguida, as estimações dos parâmetros $\left(\theta^{*}\right)$ são ajustadas de modo contínuo de tal forma que $\left(\hat{y}\left(t / \theta^{*}\right)\right)$ se aproxima de $(y(t))$ à medida que a quantidade amostral aumenta.

\section{METODOLOGIA}

\subsection{Coluna depentanizadora de nafta}

Uma unidade de tratamento de nafta foi utilizada no presente trabalho, sendo a mesma modelada na plataforma Aspen Dynamics ${ }^{\circledR}$, previamente validada com dados reais de planta de uma indústria. Para avaliar a estrutura da auto-sintonia proposta neste trabalho, uma coluna de destilação depentanizadora foi considerada, como mostrado no diagrama simplificado na Figura 3.

A coluna adotada para o estudo é constituída por 37 estágios, com uma carga de alimentação média de $57,55 \mathrm{t} \mathrm{h}^{-1}$, composta de $\mathrm{C} 1$ a $\mathrm{C} 11$ de compostos parafínicos e aromáticos mais representativos da carga real do processo. A finalidade desta coluna é separar os componentes maiores que C5 na corrente de base e os menores e iguais a C5 na corrente de destilado. 
A corrente de produto de topo, identificada como 454, é composta de 0,998 de hidrocarbonetos até o C5. A corrente do produto de base, identificada como 471, é composta de 0,999 de hidrocarbonetos maiores que o C5.

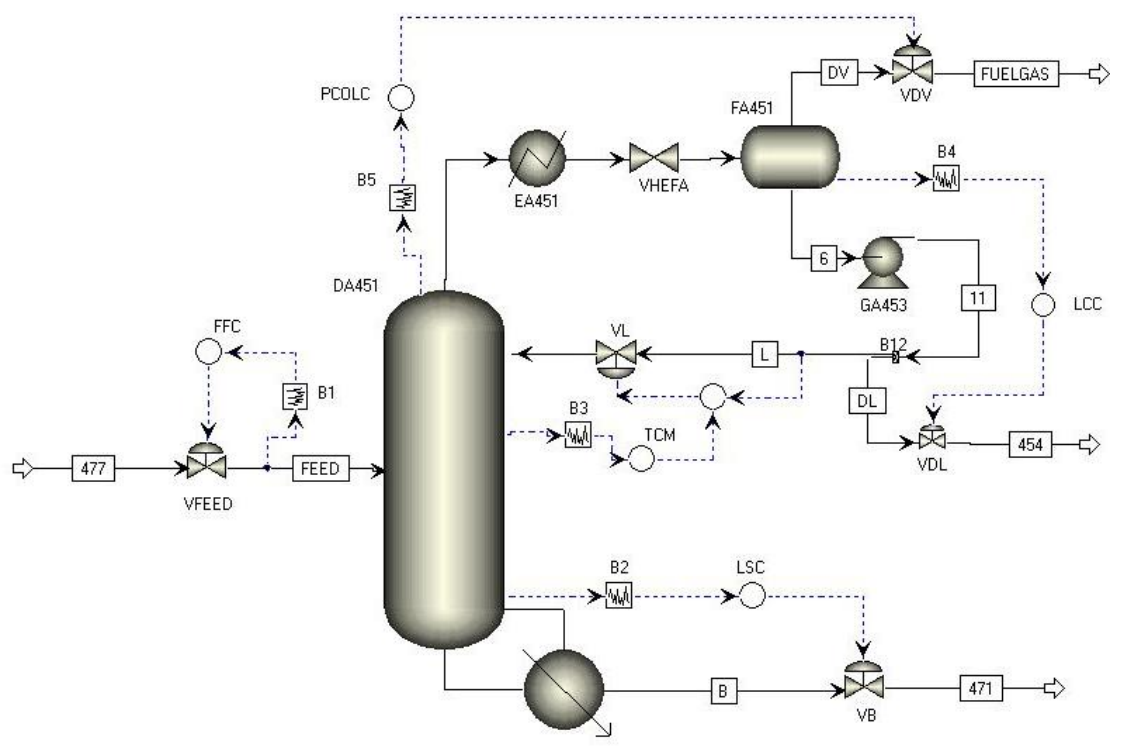

Figura 3 - Fluxograma da coluna de destilação e malhas de controle no Aspen Dynamics®.

\subsection{Algoritmo do sistema de auto-sintonia}

A partir da planta implementada na plataforma Aspen Dynamics®, a conexão com VBA foi realizada utilizando a tecnologia de comunicação Object Linking and Embedding (OLE). Dessa forma, a estimação dos parâmetros para o modelo ARMAX e a auto-sintonia foram programados em VBA considerando o algoritmo simplificado representado na Figura 4.
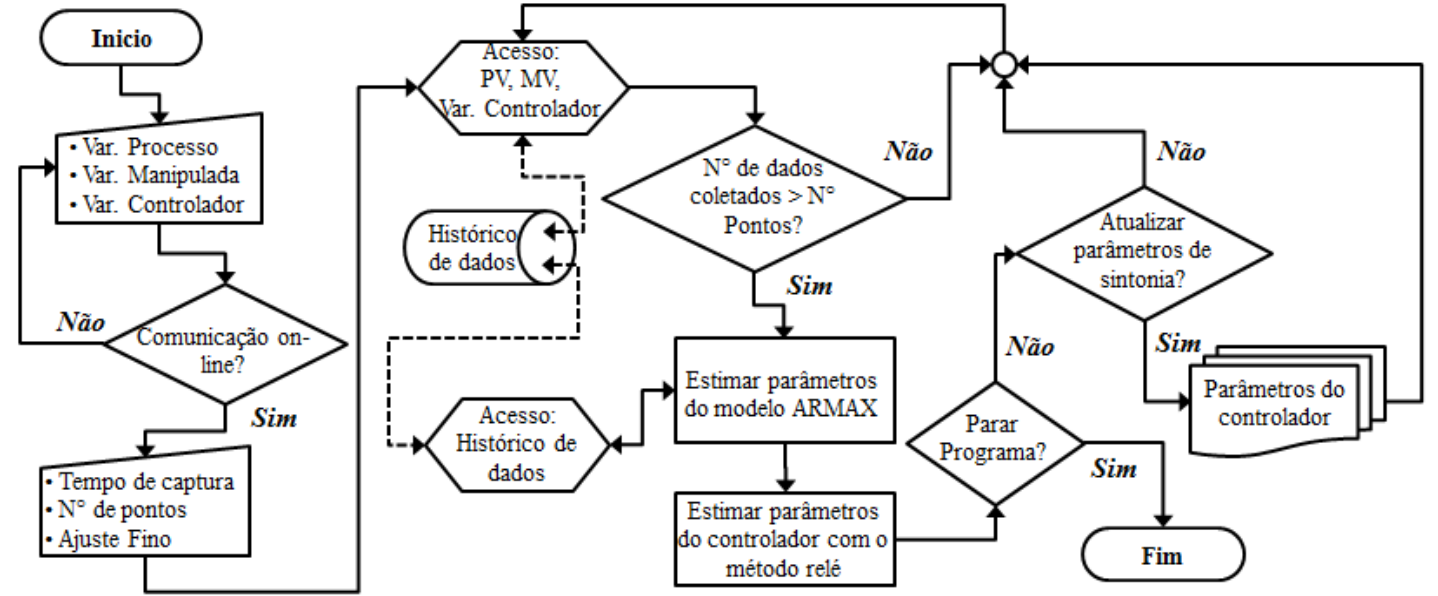

Figura 4 - Algoritmo simplificado do programa de auto-sintonia desenvolvido no VBA.

Inicialmente ocorre a entrada manual das variáveis de processo, manipulada e do controlador a serem analisadas para a malha de controle. A partir da definição dos parâmetros de tempo de captura, número de pontos e ajuste fino desejados, inicia-se o acesso às variáveis PV, MV e set-point, sendo continuamente armazenado como histórico de dados. Quando a 
quantidade de pontos coletados for superior à quantidade de pontos determinados, com acesso ao histórico de dados, será iniciado o processamento de estimação dos parâmetros pelo modelo ARMAX com uso do método dos mínimos quadrados recursivos.

Após essa etapa, utiliza-se o método do relé onde são estimados os parâmetros de sintonia do controlador PID. Ao longo do processo pode ser observado o comportamento da variável de processo simulada no Aspen Dynamics®, a variável estimada pelo modelo e o setpoint. Além disso, podem ser conferidas as modificações da variável manipulada, desvio entre valores estipulados pelo modelo e valores simulados e a determinação dos parâmetros $\boldsymbol{a}, \boldsymbol{b}$ e $\boldsymbol{c}$ do modelo ARMAX.

\section{RESULTADOS E DISCUSSÃO}

A metodologia apresentada foi aplicada nas malhas de temperatura, de vazão e de pressão. A seguir estão apresentados os resultados referentes à malha de pressão. Os resultados observados com a malha de pressão foram similares às demais malhas.

\subsection{Aplicação da ferramenta de auto-sintonia na malha de pressão}

Aplicando-se a estimação dos parâmetros do modelo ARMAX na malha de controle da pressão (PCOLC), verificou-se o comportamento no Aspen Dynamics ${ }^{\circledR}$ e a variável de processo estimada pelo modelo. Avaliou-se esse comportamento sem e com a aplicação da ferramenta de auto-sintonia e os mesmos são apresentados na Figura 5(a) e 5(b).

(a)
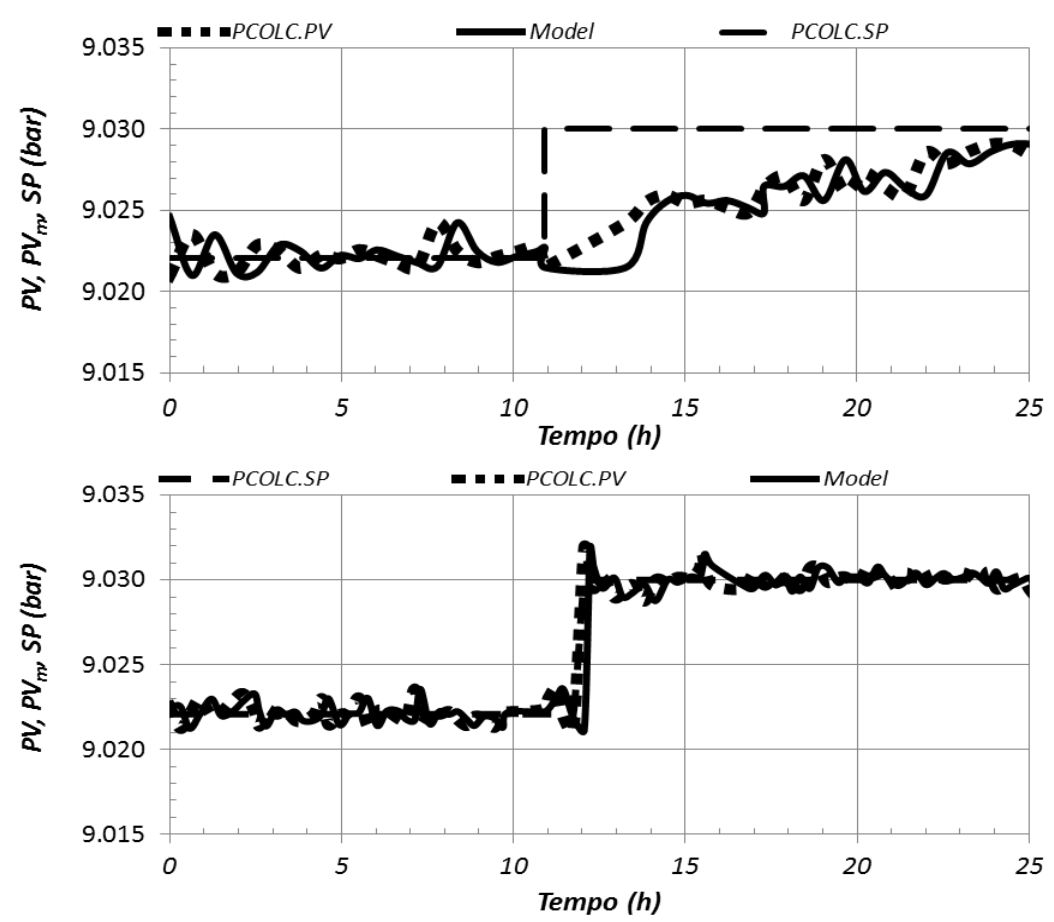

Figura 5 - Comportamento da variável de processo para malha de controle de pressão (a) sem auto-sintonia e (b) com aplicação da auto-sintonia. 
A estratégia de controle padrão dos parâmetros de sintonia do controlador PID proposta pelo Aspen Dynamics ${ }^{\circledR}$ não foi eficiente e apresentou uma resposta muito lenta e instável. O modelo, por sua vez, apresentou diferença maior em relação à PV simulada logo após o distúrbio no processo, porém foi capaz de mostrar comportamento semelhante ao simulado posteriormente. Ao aplicar a ferramenta de auto-sintonia o desempenho foi melhor, observando uma resposta mais rápida e mais estável.

Além disso, avaliou-se o desvio entre o valor da variável de processo simulada no Aspen Dynamics ${ }^{\circledR}$ e a variável estimada pelo modelo ARMAX, conforme mostrado na Figura 6. Verifica-se que o procedimento de estimação do modelo visa minimizar a diferença entre o modelo e o processo. Um desvio maior foi observado logo após o distúrbio, sendo em seguida minimizado.

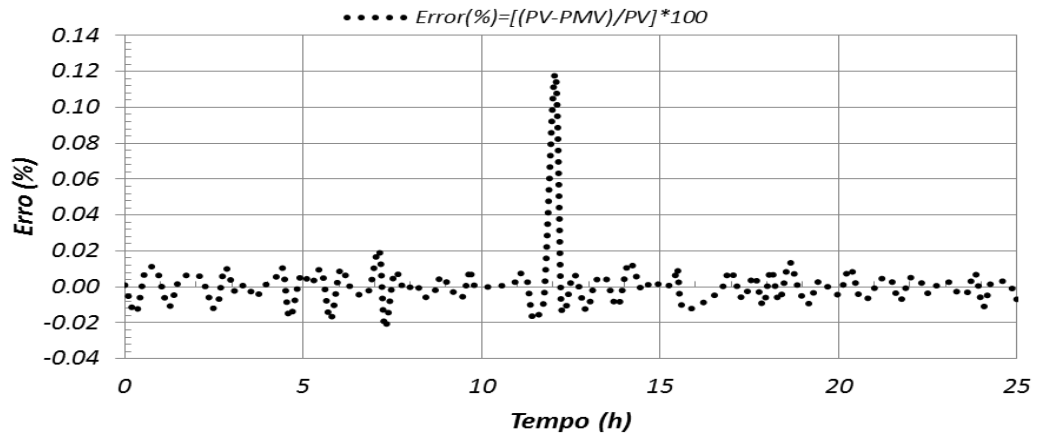

Figura 6 - Desvio entre a variável de processo simulada e variável estimada pelo modelo ARMAX para malha de controle de pressão.

Os parâmetros $(\boldsymbol{a}, \boldsymbol{b}$ e $\boldsymbol{c})$ do modelo ARMAX foram obtidos e seguem expressos na Figura 7. Alterações mínimas foram observadas para os parâmetros $\boldsymbol{a}$ e $\boldsymbol{b}$, sendo a modificação mais acentuada no parâmetro $c$ após ser inserido distúrbio, e, posteriormente, esse comportamento foi suavizado. Tais variações mostram que os mesmos são ajustados com o funcionamento do processo.
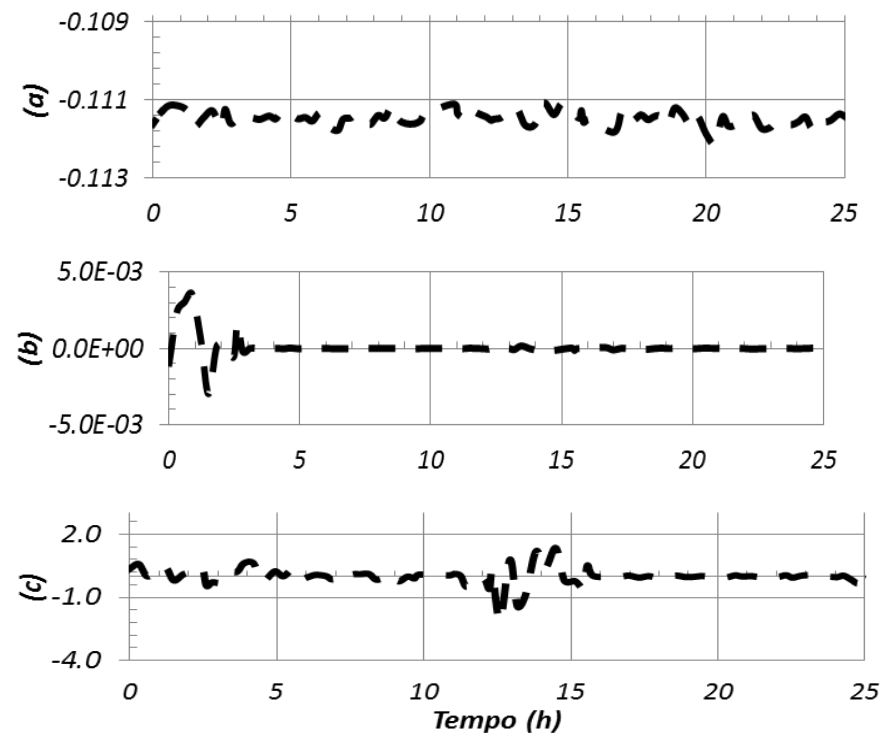

Figura 7 - Parâmetros do modelo ARMAX processo para malha de controle de pressão. 
O comportamento do posicionamento da válvula da corrente de destilado vapor, considerado como a variável manipulada, ao longo do processo é apresentada na Figura 8. Após a perturbação no processo ocorreu um fechamento da válvula da corrente de destilado vapor para tentar controlar a pressão da coluna. Após esse instante, verificaram-se mudanças em torno de $1 \%$ no posicionamento do elemento final de controle para esta malha analisada.

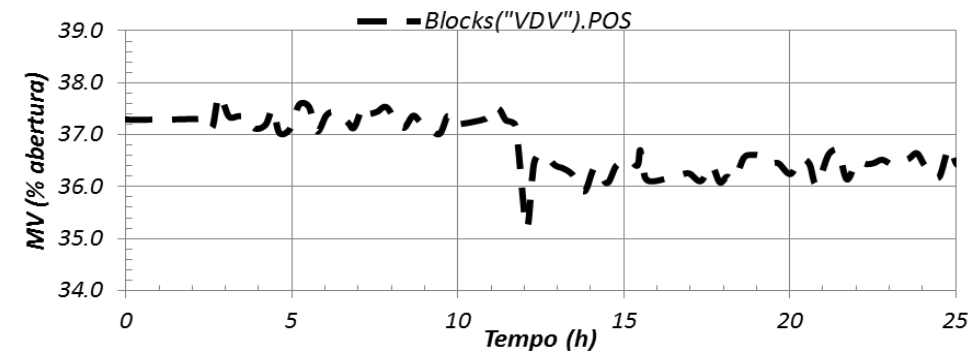

Figura 8 - Comportamento do posicionamento da válvula da corrente de destilado vapor.

\section{2 Índice de desempenho}

Para quantificar o erro ocorrido em função de uma perturbação utilizam-se critérios baseados na integral do erro. Dentre os índices baseado nos erros, utilizou-se o ITAE. Sem a auto-sintonia, o valor do ITAE foi de 1,43, enquanto que ao utilizar a ferramenta de autosintonia o valor obtido foi de 0,482 , correspondendo a uma redução $66,29 \%$. Tal redução evidencia o bom desempenho do sistema de atualização automática dos parâmetros de sintonia proposto.

\section{CONCLUSÃO}

O método do ARMAX apresentou resultados satisfatórios ao analisar o comportamento real do processo com o estimado pelo modelo. A ferramenta de auto-sintonia apresentou desempenho melhor quando comparado sem o ajuste automático dos parâmetros do controlador, e comprovou ser uma técnica bastante promissora.

\section{REFERÊNCIAS BIBLIOGRÁFICAS}

ÅSTRÖM, K. J.; HÄGGLUND, T., Automatic tuning of simple regulators with specifications on phase and amplitude margins, Automatica, v. 20, p. 645, 1984.

ÅSTRÖM, K. J., Tuning and Adaptation, IFAC $13^{\text {th }}$ Triennial World Congress, San Francisco, USA, p. 1-18,1996.

HOLST, J., Adaptive Prediction and Recursive Estimation, Department of Automatic Control, Lund Institute of Technology, Sweden, 1977.

LJUNG, L.; SÖDERSTRÖM, T., Theory and practice of recursive identification, The MIT Press (Cambridge - Massachusetts), 1983.

SILVA, J. N., Determinação da região robusta de estabilidade e de desempenho inspirada nos princípios da estatística clássica, Tese de Doutorado, Universidade Federal de Campina Grande - UFCG, 2013. 